\title{
A CHARACTERIZATION OF THE DIFFERENTIABLE SUBMANIFOLDS OF $R^{n}$
}

SAM B. NADLER, JR.

1. Introduction. It is known $\left[3\right.$, p. 49] that any class $C^{1}$ differentiable submanifold of $R^{n}$ is a (class $C^{1}$ ) differentiable neighborhood retract. In this paper we prove that the subsets of $R^{n}$ which are class $C^{1}$ neighborhood retracts (of connected open sets) are precisely the class $C^{1}$ differentiable submanifolds of $R^{n}$. In particular, Theorem 1 shows that the range of a class $C^{1}$ retraction is a class $C^{1}$ submanifold.

2. If $S$ is a linear transformation on $R^{n}$, then $\operatorname{rank}(S)$ is the dimension of the range space of $S$.

LEMмA 1. If $C$ is a connected set of idempotent linear transformations (i.e. projections) on $R^{n}$ and if $S, T \in C$, then $\operatorname{rank}(S)=\operatorname{rank}(T)$.

Proof. Let $M n$ denote the set of all real $n \times n$ matrices and let $\operatorname{Tr}: M n \rightarrow R$ be the trace operator, i.e., $\operatorname{Tr}(A)=\sum_{i=1}^{n} a_{i i}$ where $A=\left(a_{i j}\right)$. It is easily verified that $\operatorname{Tr}$ is continuous on $M n$ and an invariant of similarity class $[2$, p. 96]. Suppose that $A \in M n$ is an idempotent. Then $A$ is similar to a matrix $B=\left(b_{i j}\right)$ such that $b_{i i}=1$ for $1 \leqq i \leqq \operatorname{rank}(A)$ and $b_{i j}=0$ otherwise. Hence, $\operatorname{Tr}(A)=\operatorname{Tr}(B)$ $=\sum_{i=1}^{\mathrm{rank}(\overline{\mathrm{A}})} b_{i i}=\operatorname{rank}(A)$. Letting the trace of a linear operator be the trace of any matrix representation, it follows that the trace of any member of $C$ is its rank and, therefore, that $\operatorname{Tr}$ is constant on $C$. Hence, $\operatorname{Tr}(S)=\operatorname{Tr}(T)$, i.e., $\operatorname{rank}(S)=\operatorname{rank}(T)$.

If $S$ and $T$ are projections having the same rank $r$, then there is an arc of projections of rank $r$ joining $S$ and $T$. It now follows from Lemma 1 that, in the space of linear transformations on $R^{n}$, there are precisely $n+1$ components of idempotent linear transformations, two idempotents being in the same component if and only if they have the same rank.

The proof of the next lemma may be found in [1, pp. 273-276].

Lemma 2 (RANk Theorem). Let $E$ be an $n$-dimensional space, $F$ an $m$-dimensional space, $A$ an open neighborhood of a point $a \in E, f a$ continuously differentiable mapping of $A$ into $F$, such that in $A$ the rank of $f^{\prime}(x)$ is a constant number $p$. Then there exists

1. an open neighborhood $U \subset A$ of $a$, and a homeomorphism $\mu$ of $U$ onto the open unit $n$-cube $I^{n}=\left\{\left(x_{1}, x_{2}, \cdots, x_{n}\right) \in R^{n}:\left|x_{i}\right|<1\right.$ for

Received by the editors December 6, 1965. 
$i=1,2, \cdots, n\}$ which is of class $C^{1}$ as well as its inverse;

2. an open neighborhood $V \supset f(U)$ of $b=f(a)$, and a homeomorphism $\nu$ of the open unit $m$-cube $I^{m}=\left\{\left(x_{1}, x_{2}, \cdots, x_{m}\right) \in R^{m}:\left|x_{i}\right|<1\right.$ for $i=1,2, \cdots, m\}$ onto $V$ which is of class $C^{1}$ as well as its inverse; such that $f=\nu \circ f_{0} \circ \mu$, where $f_{0}: I^{n} \rightarrow I^{m}$ is given by $f_{0}\left(x_{1}, x_{2}, \cdots, x_{n}\right)$ $=\left(x_{1}, x_{2}, \cdots, x_{p}, 0, \cdots, 0\right)$.

THEOREM 1. Let $U$ be a connected open subset of $R^{n}$ and let $f: U \rightarrow U$ be a class $C^{1}$ retraction of $U$. If $r=\operatorname{rank}\left(f^{\prime}(x)\right)$, for some $x \in f(U)$, then $f(U)$ is a class $C^{1}$ differentiable submanifold of $R^{n}$ of dimension $r$.

Proof. We first show that the rank of $f^{\prime}(z)=r$ for each $z \in f(U)$. To see this, let $z \in f(U)$. Since $f(z)=z, f^{\prime}(z)=(f \circ f)^{\prime}(z)=f^{\prime}(f(z)) \circ f^{\prime}(z)$ $=f^{\prime}(z) \circ f^{\prime}(z)$ and, therefore, $f^{\prime}(z)$ is an idempotent linear transformation. Since $f(U)$ is connected and $f^{\prime}$ is continuous, $f^{\prime}(f(U))$ is a connected set of idempotents. Therefore, by Lemma 1, rank $\left(f^{\prime}(z)\right)$ $=\operatorname{rank}\left(f^{\prime}(x)\right)=r$.

Now let $p \in f(U)$. Since rank $\left(f^{\prime}(p)\right)=r$, there is a set $V$, open in $R^{n}$ and containing $p$, such that $r \leqq \operatorname{rank}\left(f^{\prime}(y)\right)$ for all $y \in V$. However, if $y \in V$, then $\operatorname{rank}\left(f^{\prime}(y)\right)=\operatorname{rank}\left((f \circ f)^{\prime}(y)\right)=\operatorname{rank}\left(f^{\prime}(f(y)) \circ f^{\prime}(y)\right)$ $\leqq$ rank $\left(f^{\prime}(f(y))\right)=r$ since $f(y) \in f(U)$ and since the rank of the composition of two linear transformations is less than or equal to the rank of each. This shows that rank $\left(f^{\prime}(y)\right)=r$ for all $y \in V$. By Lemma 2, there is

1. a set $W \subset V$, open in $R^{n}$ and containing $p$, and a homeomorphism $g$ of $W$ onto the open unit $n$-cube $I^{n}$ such that both $g$ and $g^{-1}$ are of class $C^{1}$;

2. a set $W^{\prime} \supset f(W)$, open in $R^{n}$ and containing $f(p)=p$, and a homeomorphism $h$ of the open unit $n$-cube $I^{n}$ onto $W^{\prime}$ such that both $h$ and $h^{-1}$ are of class $C^{1}$; such that $f=h \circ f_{0} \circ g$, where $f_{0}: I^{n} \rightarrow I^{n}$ is given by $f_{0}\left(x_{1}, x_{2}, \cdots, x_{n}\right)=\left(x_{1}, x_{2}, \cdots, x_{r}, 0, \cdots, 0\right)$.

If $H=h^{-1}$, then $\left(W^{\prime}, H\right)$ is a coordinate system of $R^{n}$ such that $H\left(W^{\prime} \cap f(U)\right)=h^{-1}\left(W^{\prime}\right) \cap h^{-1}(f(U))=h^{-1}(f(U))=f_{0}(g(U))=f_{0}\left(I^{n}\right)$ $=I^{r}$, where $I^{r}=\left\{\left(x_{1}, x_{2}, \cdots, x_{n}\right) \in I^{n}: x_{i}=0\right.$ for $\left.r<i \leqq n\right\}$ is an open subset of $R^{r}$. Hence, there is a covering of $f(U)$ by coordinate systems $\left(W^{\prime}, H\right)$ of $R^{n}$ such that $H\left(W^{\prime} \cap f(U)\right)$ is an open subset of $R^{r}$. This proves that $f(U)$ is a class $C^{1}$ differentiable submanifold of $R^{n}$ of dimension $r$, as defined in [3, p. 15].

The following theorem characterizes the class $C^{1}$ submanifolds of $R^{n}$ as class $C^{1}$ neighborhood retracts.

Theorem 2. A subset $M$ of $R^{n}$ is a class $C^{1}$ differentiable submanifold of $R^{n}$ if and only if there is a connected set $W \supset M, W$ open in $R^{n}$, and a class $C^{1}$ retraction $f$ of $W$ onto $M$. 
Proof. The sufficiency is Corollary 5.6 in $[3$, p. 49]. The necessity is a simple application of Theorem 1 of this paper.

It would be interesting if Theorem 1 could be generalized to Banach spaces (and Banach manifolds). The main problem seems to be an appropriate general form of the Rank Theorem. The author has some theorems which show that the range of a class $C^{1}$ retraction (not the identity function) of a connected open subset of a Banach space is nowhere dense.

The results in this paper extend immediately to class $C^{1}$ manifolds. The author wishes to express his appreciation to Professor John Jewett for his helpful suggestions concerning the material in this paper.

\section{REFERENCES}

1. J. Dieudonné, Foundations of modern analysis, Academic Press, New York, 1961.

2. K. Hoffman and R. Kunze, Linear algebra, Prentice-Hall, Englewood Cliffs, N. J., 1964.

3. J. R. Munkres, Elementary differential topology, Princeton Univ. Press, Princeton, N. J., 1963.

UNIVERSITY OF GEORGIA AND

Wayne State University 